\title{
COVID 19 and Psychiatric Morbidity
}

The COVID 19 virus was first identified in Wuhan in China in 2019. It spread to the entire world subsequently and gave rise to a global pandemic. Although the virus is primarily known to cause respiratory symptoms, there are instances of gastrointestinal symptoms as well as neurological sequelae. The main route of neurotropism is ACE2 receptors expressed in neurons and neuroglia. The SARS-CoV-2, similarly to other respiratory viruses, could gain access to CNS through several routes, for example by migrating through axons of the olfactory nerve.[1] The virus infects and damages the brainstem where respiratory center is located. The inflammatory response, so commonly described in COVID 19 infections, also damages the blood brain barrier causing further destruction to central nervous system through inflammatory chemicals. These massive changes to the brain milieu may be responsible for various neuropsychiatric sequelae like delirium, dementia, stroke as well as form the organic basis of psychiatric conditions like major depressive disorder, anxiety disorder and reactive psychosis. Similarly, massive psychosocial upheavals caused by the overwhelming global pandemic including change in lifestyle and socialization, being forced to stay indoors for weeks together due to lockdowns, financial consequences of unemployment and downslide of economy have all lead to increase in anxiety, depression and even suicidality. This is bound to increase further as time passes and economy takes a further beating. Prolonged stay in intensive care units, fear of imminent death, loss of loved ones all of a sudden- all these have caused increased incidences of post traumatic stress disorder. Overall, the COVID 19 is poised to increase the burden of neuropsychiatric conditions manifold. Longitudinal studies must be undertaken to gain clarity and further understanding on the neuropsychiatric sequelae of COVID 19 and their mechanism.

\section{Dr. Sujit Sarkhel Hony. Editor}

\section{REFERENCES}

1. Desforges, M., Le Coupanec, A., Brison, É., Meessen-Pinard, M. \& Talbot, P. J. Neuroinvasive and neurotropic human respiratory coronaviruses: potential neurovirulent agents in humans. Adv. Exp. Med Biol. 807, 75-96 (2014). 